\title{
Methodological examination of the PEST (Parametric Estimation by Sequential Testing) procedure
}

IRWIN POLLACK

MENTAL HEALTH RESEARCH INSTITUTE, UNI'ERSITY OF MICHIGAN, ANN ARBOR

Any psychophysical procedure requires arbitrary dec isions on the part of $E$ at the beginning of the experiment. Even within adaptive stimulus programming, e.g., PEST, procedures, which are modified according to the moment-by-moment responses of $S, E$ must initialize the procedure. The PEST procedure was employed in three different auditory tasks and examined for its immunity to initializing conditions. PEST, despite its adaptive features, is not entirely immune to initializing conditions. It is suggested that an iterative approach may assist the initializing of conditions for psychophysical procedures.

Psychophysical procedures are designed to provide answers to questions posed by the psychophysical investigator. When $E$ is exploring, he does not wish to formulate his question prematurely, and he would be wise to employ procedures which would give him the widest view of the landscape (Broadbent, 1957). After the landscape is surveyed, $\mathrm{E}$ may be able to ask more specific questions, such as "Given the values $i, j, k$, for variables $u, v, w$, what is the level, $l$, for variable $x$ which will yield $75 \%$ correct in a fourinterval forced-choice procedure?" When the question becomes sufficiently well-defined, it becomes possible to introduce efficient psychophysical procedures which, for the given criterion of "accuracy," will yield an answer with a smaller investment of time (Cornsweet, 1962; Smith, 1962). Except for purists who worship traditional methods, as such (Dallenbach, 1967), few would argue with the contention that efficiency is a desirable aim in its own right. There is, however, a price that must be paid with any efficient psychophysical procedure: serendipity is replaced by efficiency. For example, if a specific phenomenon is obtained only at near-perfect performance levels, that phenomenon might well be missed by an efficient psychophysical procedure which converges upon a $75 \%$ correct performance level.

Smith (1961, p. 30) also pointed out that a valuable extension to existing efficient psychophysical procedures would be a method for changing the size of the experimental variable as the experiment proceeds. When $E$ is uncertain of where S's threshold lies, he should explore in giant-size steps; when the threshold region becomes better defined, $E$ should explore in small-size steps.

The feature of a variable step size which parallels
E's uncertainty is incorporated into a new psychophysical procedure-PEST (Parameter Estimation by Sequential Testing, Taylor \& Creelman, 1967). The PEST procedure is attractive because of the following properties: (1) The procedure is well-defined. The rules are completely specified. The burden of running $\mathrm{S}$ can, therefore, be turned over to a computer. (2) It provides an efficient point estimate of S's threshold. Taylor and Creelman (1967) provide an extensive discussion of the efficiency of PEST. (3) Embodied in the procedure is the rule when to stop collecting data. The $S$ need not dwell longer than necessary upon any one condition.

The combination of the three properties make possible a truly efficient automated laboratory (Pollack, Headly \& Maas, 1966). Our experimental $S$ identifies himself to a PDP -8 computer (Digital Equipment Corporation); he identifies the starting trial (based upon a record of previous work), and he identifies the direction he wishes to proceed through the list of experimental conditions. Thereafter, the computer takes over completely. The E's main role is to describe the task initially for $S$. However, a single instruction works for all four-interval forced-choice tasks: select that one signal which sounds different from the other three. The net effect is to cut down covert communication between $\mathrm{E}$ and $\mathrm{S}$ (Rosenthal, 1967).

PEST, like all psychophysical procedures, requires $\mathrm{E}$ to make arbitrary, but necessary, decisions in initially setting up his experiments. What are the effects of these decisions upon the final experimental results? A good psychophysical procedure should "protect" experimental data from arbitrary decisions made by $E$ (Pollack, 1965). If the answer yielded by the PEST procedure is sensitive to $E^{\prime} s$ arbitrary decisions, the procedure is a poor procedure, regardless of its efficiency. If the answer yielded by the PEST procedure is insensitive to such decisions, the procedure may be expected to lead to fewer biased results, in addition to its efficiency. In short, the present tests attempt to determine some pragmatic rules-of-thumb for using the PEST procedure for some specific auditory psychophysical situations. No claim is made for the generality of the rules to other situations, except to note that the procedure has been satisfactorily employed in a wide variety of auditory, visual, and vibrotactile tasks in our laboratory. 


\section{METHOD}

\section{Rules}

The rules in our application of the PEST procedure are schematically represented in Fig. 1. The description will be in terms of stimulus differences for a Yes-No experiment. The forced-choice equivalent substitutes "correct" for "yes" and "incorrect" for "no."

1. Reversal rule. The magnitude of the change in stimulus magnitude between successive stimulus presentations-here called the step size-is halved upon each reversal, as in obtaining a yes response after a no response, or vice versa.

2. Exit rule. If the step size upon the next trial is less than an arbitrary pre-set exit criterion, the trial is terminated. The value of the stimulus on the next trial is the point estimate of its threshold.

3. Doubling rule. The step size is doubled upon the occurrence of three successive yes, or three successive no, responses. Otherwise, the step size is the same as the previous step size. Taylor and Creelman (1967, pp. 783-4, Rule 4) employ a somewhat more complex rule. The departure from their rule is expected to provide only a small loss of efficiency without a bias of the mean.

4. Maximum rule. The maximum size step is limited to a constant multiplied by the exit criterion.

5. Initial rule. The size of the initial stimulus difference is set to a constant multiplied by the exit criterion. In order that a decision can be made to the response following the first stimulus presentation, it is necessary to assume the step size and the direction for two (imaginary) presentations prior to the first actual stimulus presentation.

6. "Bumping into zero" rule. If the step size will modify the size of the stimulus difference to a difference below zero, the step size is successively halved

\section{PEST RULES}

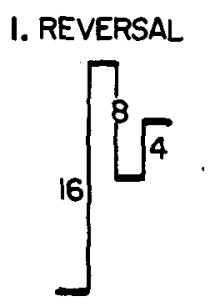

$$
\text { 2. EXIT }
$$

3. DOUBLING
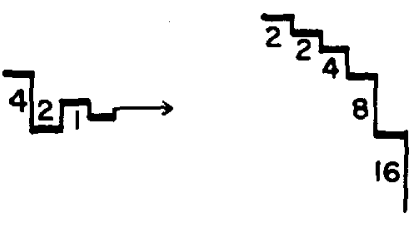

4. MAXIMUM

5. INITIAL
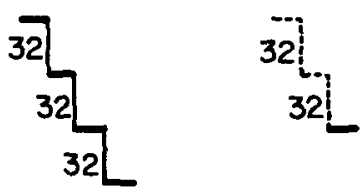

Fig. 1. Schematic illustration of rules of PEST procedure (see text). until the difference is greater than zero. Specifically, if the stimulus difference is 31 and a reduction of 32 is called for, a difference of -1 would have resulted. Instead, a reduction of 16 is instituted with a resulting difference of 15 . On the next trial, if a reduction of 16 is called for, it is rejected and a reduction of 8 is instituted, etc.

\section{Arbitrary Specifications}

Although the rules are well-defined, it is noted that $E$ initially must specify at least six arbitrary properties: (1) the exit criterion (EC); (2) the maximum step size, which is, in turn, a function of EC; (3) the initial stimulus difference (ISD); (4) the step size assumed to be in force for the two trials previous to the first stimulus presentation; (5) the direction of change of the step size assumed to be in force for the two trials previous to the first stimulus presentation; (6) the experimental procedure.

Independent manipulation of each of the five parameters did not seem advisable. It was guessed that the principal variables were the size of the EC, the exit criterion, and ISD, the initial starting difference. The maximum step size was permitted to co-vary with the exit criterion at 32 times the exit criterion. The initializing conditions assumed that in the two trials previous to the initial presentation, $S$ had responded correctly at the maximum step size. And, finally, Experiment 1 employed a four-interval forcedchoice procedure throughout.

One other arbitrary decision was introduced. In order to maximize use of the bumping-into-zero rule, ISD was selected at $2^{\mathrm{n}}$ times $\mathrm{EC}$ minus one. The effect of this rule is to avoid premature termination of the test sequence. For example, with ISD $=32 \mathrm{EC}$ a reduction of $32 \mathrm{EC}$ would prematurely terminate the experiment at zero difference. As shown in Fig. 1 an ISD of 31 EC would permit five successive successes before termination of the test sequence.

\section{Implementation}

The computer generated the stimulus patterns, carried out the experiment according to PEST, and terminated the experiment according to PEST. Brief pulses, $10 \mu \mathrm{sec}$, before spread out by Koss PRO-4 earphones, were presented at a comfortable listening level.

In Experiment 1, a four-alternative forced-choice version of Uttal and Krissoff's (1966) "gap" test was employed. Four pulse trains were presented to $\mathrm{S}$ : three "standard" pulse trains which, within a given trial, were constant; and a "variable" pulse train which was under the control of the PEST procedure. The variable train was identical to the standard, except that a temporal gap was introduced into the intermediate portion of the train.

Two gap problems were examined. The problems were chosen to yield widely different answers. Except 
for the gap, the interval between successive pulses was equal to about $2 \mathrm{msec}(1.9 \mathrm{msec}$ to be more precise). In the first problem, one interval preceded and followed the gap. The standard consisted of the following interpulse intervals: $2,2,2 \mathrm{msec}$; the variable consisted of $2,2-\Delta, 2 \mathrm{msec}$. In the second problem, two interpulse intervals surrounded the gap interval and the entire pattern was repeated 16 times. Specifically, the variable consisted of the following intervals: 2,2 , $2-\Delta, 2,2 \mathrm{msec}$, repeated 16 times. The standard consisted of 80 intervals, each $2 \mathrm{msec}$ long.

With the first gap problem, EC varied from 1 to 256, and ISD varied from 63 to 1023 temporal units, each $0.375 \mu \mathrm{sec}$. This yielded 21 combinations of EC and ISD. Eleven trained Ss with an average of $50 \mathrm{~h}$ of previous experience were employed. They collectively contributed 19-31 thresholds for each condition (median: 28 thresholds).

With the second gap problem, the exit criterion varied from 1 to 64 temporal units, and the initial starting difference varied from 3 to 512 temporal units. This yielded 35 combinations of EC and ISD. Six trained Ss, with an average of about $50 \mathrm{~h}$ of previous experience were used in the tests. Each condition is represented by approximately 21 tests: four Ss each completed four tests per condition; one $S$ each completed three and two tests per condition, respectively. Estimates of median within-S variance were obtained from the four Ss who completed four tests per condition.

In Experiment 2, three two-alternative procedures were employed in conjunction with a glissando interval discrimination. In such a test, the successive intervals change either in an ascending direction or in a descending direction. Thus, for five intervals in one direction, successive intervals of the pulse train would be $A+2 \Delta, A+\Delta, A, A-\Delta, A-2 \Delta$, where $A$ is the average interval and $\Delta$ is the constant change per interval. In the opposite direction, successive intervals of the pulse train would be: $A-2 \Delta, A-\Delta, A, A+\Delta, A+2 \Delta$.

Three procedures were examined. In a same-different procedure, two pulse trains were presented successively. On half of the trials, the direction of change of the pulse trains was different; on half of the trials the direction was the same. S's task was to respond same or different. In a one-interval forced-choice procedure, one pulse train was presented. S's task was to indicate whether the glissando was upward or downward. In a two-interval forced-choice procedure, two pulse trains were presented in which the direction of glissando differed. S's task was to indicate the direction of glissando of the first train. Each of five Ss completed one test under each of the testing conditions. It is noted that the Ss had extensive previous experience with all procedures. PEST tracked $75 \%$ correct response in the two-alternative test according to the following rules. In a defined trial-block of four successive trials, $\Delta$ decreased if $S$ made four correct responses out of four trials; $\Delta$ increased if $S$ made two incorrect re-

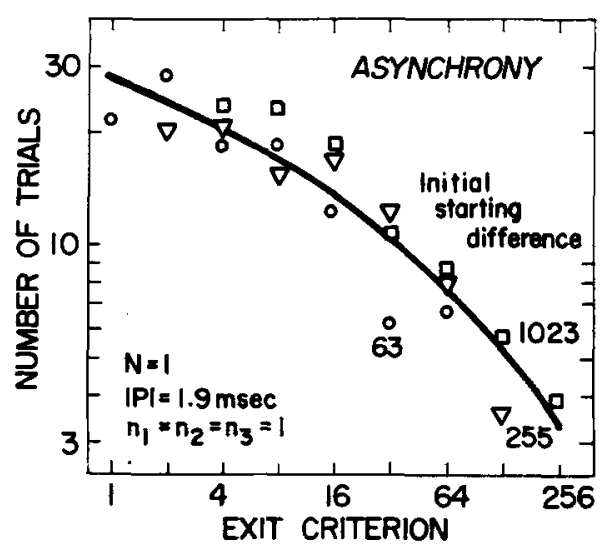

Fig. 2. The number of trials required to satisfy the PEST exit criterion in an asynchrony discrimination task as a function of the initial starting difference and the magnitude of the exit criterion.

sponses out of two, three or four trials, and $\Delta$ did not change if $S$ made three correct responses out of four trials.

\section{Experiment 1}

\section{RESULTS}

Figures 2 (first gap test) and 3 (second gap test) examine the effect of EC and ISD upon the average number of forced-choice trials required before exiting in the PEST procedure. The number of trials is highly sensitive to the exit criterion-the "grain" of the observations. This is reasonable: one should expect to obtain a ballpark estimate faster than a finer estimate. The number of trials is relatively insensitive to the initial starting difference except for extreme conditions. In this connection, it is noted that the bumping-into-zero rules will permit exiting after $n$ successive successes when ISD $=\left(2^{n} \mathrm{EC}-1\right)$. Thus, by chance alone, small ratios of ISD to EC will yield an answer in a spuriously small number of trials. Difficulties may occur when the ratio of (ISD+1) to EC is less than eight.

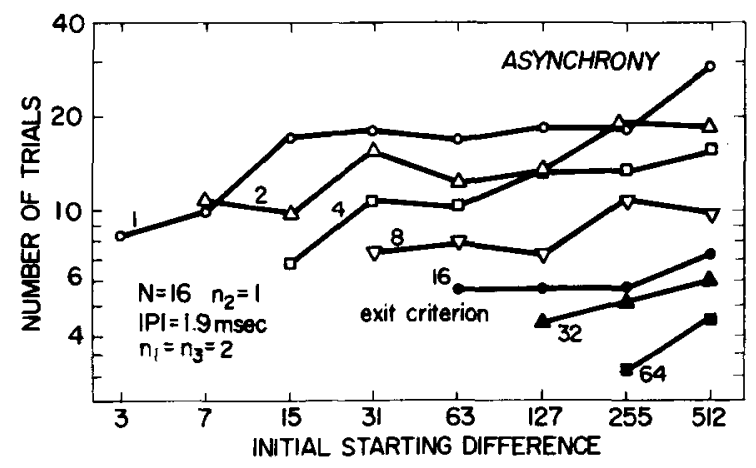

Fig. 3. As Fig. 2 with a different asynchrony task. 


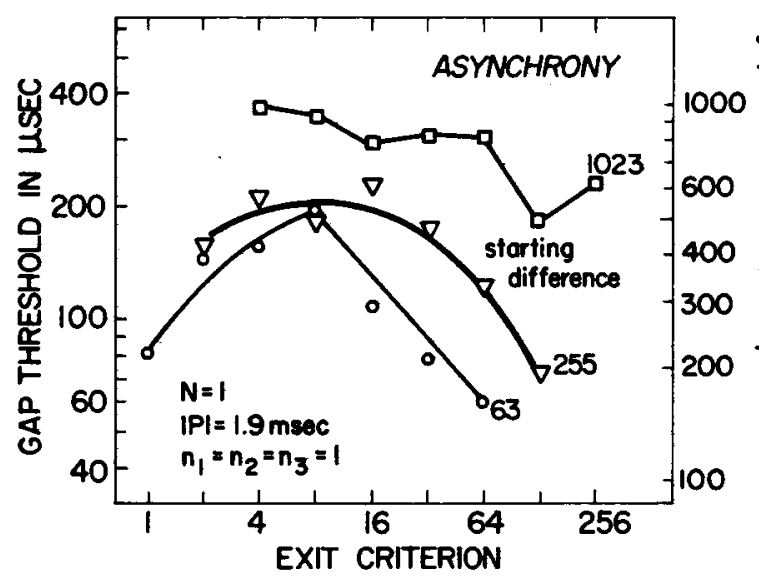

Fig. 4. Average gap thresholds yielded by PEST as a function of the initial starting difference and of the exit criterion.

The corresponding average gap thresholds yielded by the PEST procedure are presented in Figs. 4 (first gap test) and 5 (second gap test). In terms of the median gap threshold over all conditions, the first gap test of Fig. 4 yields a ballpark estimate of 540 temporal units; the second gap test of Fig. 5 yields a ballpark estimate of 45 temporal units.

Relatively large effects are observed upon the mean gap threshold. In Fig. 4, over all conditions, the average PEST threshold varies over a 6-fold range. If we observe the restriction that (ISD +1$) \geq 8 \mathrm{EC}$, then the range of thresholds is cut in half (with the exception of one condition).

Experimental conditions are represented in Fig. 5 in two ways. In the left panel the abscissa is the exit criterion, and the parameter represents the initial stimulus difference; in the right panel the abscissa is the ratio of IDS to EC. Again, a large range of scores is obtained over all conditions. The right panel suggests that when ISD +1$) \geq 32 \mathrm{EC}$, the 7 -fold range of scores shrinks to a 2 -fold range of scores.

Figure 6 considers the median intra-S variance.

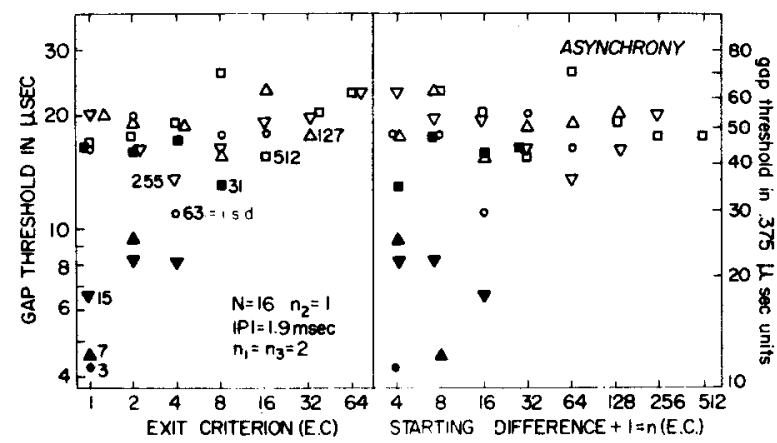

Fig. 5. As Fig. 4 (loft section) with a different asynchrony task. The right section replots the points of the left section in terms of the ratio of the initial starting difference to the exit criterion

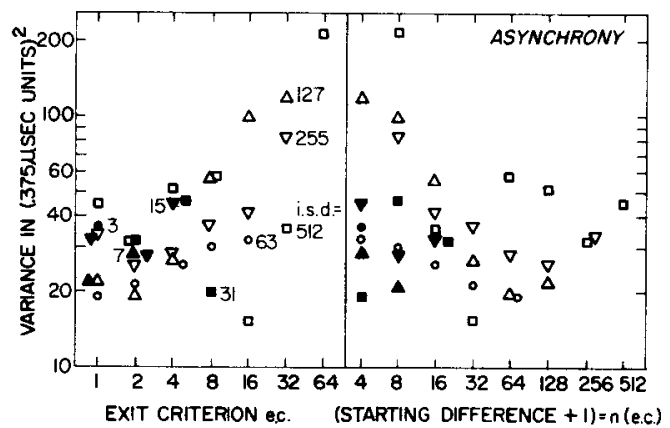

Fig. 6. Median within-subject variance in the gap threshold as a function of the exit criterion (left section). The right section replots the points of the left section in terms of the ratio of the initial starting difference to the exit criterion.

The organization of Fig. 6 parallels that of Fig. 5 . Again, a large range of scores is obtained over all conditions. The right panel suggests that when ISD $\geq 16 \mathrm{EC}$, a 16-fold range of variance scores shrinks to a 4.5 -fold range of scores.

\section{Experiment 2}

Figure 7 presents the results of the three procedures over a wide range of interpulse intervals (abscissa) and number of pulses (parameter). Although there are minor differences in results among the conditions, the general picture which emerges is quite similar across all three procedures, except under

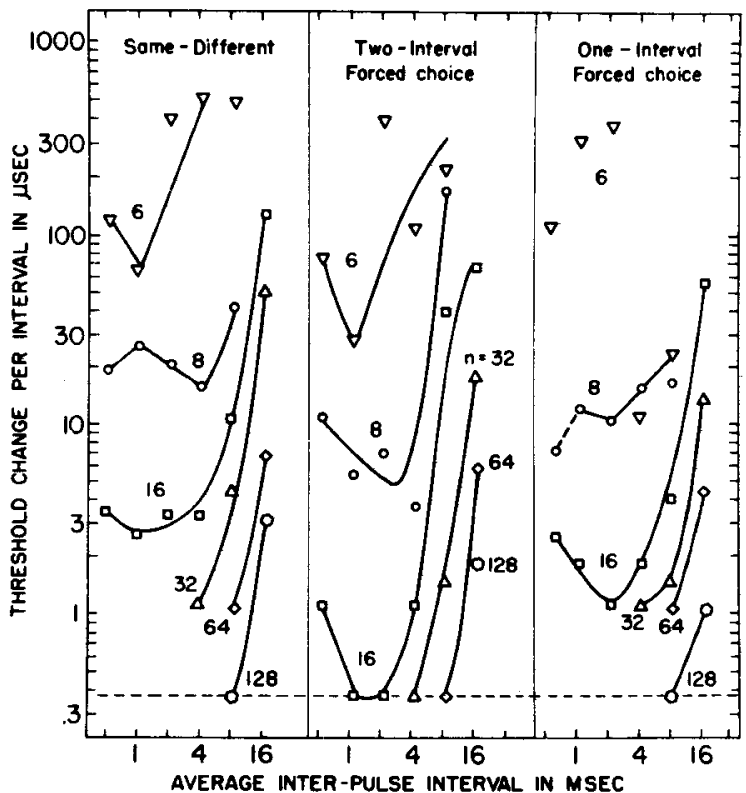

Fig. 7. Comparison among three psychophysical procedures each employing the PEST procedure. The parameter on the curves is the number of pulses in a glissando pulse sequence. 
the marginally discriminable condition of five intervals (six pulses).

\section{DISCUSSION}

The results of Experiment 1 suggest that PEST is not immune to arbitrary decisions made by $E$ at the beginning of the experiment. The resuits of Experiment 2 suggest that the interactions between PEST rules and the specific experimental technique may be small. Experience has suggested that informal preliminary experimentation is important in judiciously selecting initial conditions '(see also Stevens, 1955), that $S$ prefers easy discrimination trials at the beginning of an experimental condition in order to better define his task, and that an extremely fine exit criterion may be selfdefeating. The latter statements imply that ISD should initially be moderately large. We typically aim to obtain an estimate of threshold within 12-14 fourinterval forced-choice trials. We approach this aim by setting ISD about 3 times the median expected threshold value with ISD equal to $31 \mathrm{EC}$. This procedure then employs an exit criterion of the order of $10 \%$ of the measured threshold. While the grain of the experiment is coarse, these conditions impose a relatively low bias and provide an answer in a relatively small number of trials.

\section{References}

Broadbent, D. E. Perception and communication. New York: Pergamon Press, 1958.

Cormsweet, T. N. The staircase-method in psychophysics. Amer. J. Psychol., 1962, 75, $₫ 85-491$

Dallenbach, K. M. The staircase-method critically examined. Amer. J. Psychol., 1966, 79, 654-656.

Pollack, I. Iterative techniques for unbiased rating scales. Quart. J. exp. Psychol., 1965, 17, 139-148.

Pollack, I., Headly, P., \& Maas, E. Modest Computer controlled psychoacoustical facility. J. Acoust. Soc. Amer., 1966, 39, 1248 (A).

Rosenthal, R. Covert communication in the psychological experiment. Psychol. Bull., 1967, 67, 356-367.

Smith, J. E. K. Stimulus programming in psychophysics. Psychometrika, 1961, 26, 27-34.

Stevens, S. S. On the averaging of data. Science, 1955, 121, 113116.

Taylor, M. M., \& Creelman, C. D. PEST: Efficient estimates on probability functions. J. Acoust. Soc. Amer., 1967, 41, 782-787.

Uttal, W. R., \& Krissoff, M. Effect of stimulus pattern on temporal acuity in the somatosensory system. J. exp. Psychol, 1966, 71. 878-883.

(Accepted for publication December 1. 1967)

\section{Erratum}

Curtis, D. W., Attneave, F., and Harrington, T. L. A test of a two-stage model of magnitude judgment. Percept. and Psychophys., 1968, 3, 25-31. In addition to the misspelling of "'logarithm" in a footnote, this paper contains several typographical errors: (1) The last sentence of the abstract is a telescoping of the following two sentences. "Individual values of the inferred sensory exponent, $k$, show significantly better uniformity over Os than do values of the phenotypical magnitude exponent $\mathrm{n}$. The average value of $\mathrm{k}$ is almost identical with the exponent previously found to describe interval judgments of weights." (2) The missing standard error in Table 1 (associated with the value .746) is .032. (3) In Table 1 and elsewhere, estimates of the output exponent from the quotient $\mathrm{n} / \mathrm{k}$ should be designated $\widetilde{\mathrm{m}}$; thus the expression on p. 29, col. 2, line 12 should be $1 \mathrm{~m}$

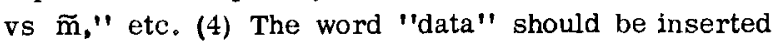
into the last sentence of Note 3, thus: "... the power model would have to fit data like..." 